\title{
Treatment of venous ulcers in drug addicts: A case report
}

\author{
Mirela Vasileva', Vesna Brishkoska Boshkovski ${ }^{2}$, Andrej Petrov ${ }^{3,4}$, Elizabeta Zisovska ${ }^{4}$ \\ ${ }^{1}$ Clinical Hospital Shtip, North Macedonia, ${ }^{2}$ City General Hospital " 8 th September" Skopje, North Macedonia, ${ }^{3}$ Acibadem \\ Sistina Hospital, Skopje, North Macedonia, ${ }^{4}$ University “Goce Delchev” Shtip, North Macedonia
}

Corresponding author: Mirela Vasileva, MD, E-mail: mirelanaceva@yahoo.com

\begin{abstract}
Venous ulcers are common in drug addicts and, although the management of these wounds is the same as in other patients, there are differences in the approach and the outcome of treatment. Those injecting drugs are at risk of serious infections, such as necrotizing fasciitis, wound botulism, and cutaneous anthrax due to the nature of the substances being injected. Herein, we present two cases of venous ulcers in drug addicts in their thirties. Both patients had been suffering from a venous ulcer for several years and the final result differed in the two patients. We concluded that it is necessary to raise awareness of the importance of treatment and lifestyle changes. The multidisciplinary approach in these patients may contribute to the improvement of wound healing.
\end{abstract}

Key words: Drug addiction; Venous ulcer; Wound healing

\section{INTRODUCTION}

Venous ulcers are common in drug addicts and, although the management of these wounds is the same as in other patients, there are differences in the approach and the outcome of treatment. Patients with a history of intravenous drug use are affected by numerous risk factors for developing chronic venous insufficiency due to damage to superficial veins through repeated trauma and thrombophlebitis.

The reason for the appearance of venous ulcers in these individuals is of course the injection of narcotics in the femoral vein, which leads to deep vein thrombosis. Repeated injections and additional damage to the venous wall cause poor blood flow, which, in combination with inactivity, emphasizes preexisting venous insufficiencies.

The preference for injection into the femoral vein is usually due to its size and easy availability. Over time, the vein becomes scarred and thickens, making it difficult to puncture, thus the addict is forced to employ thicker needles, which further damages the vein wall [1].

Through reinjection, the lumen of the femoral vein narrows, creating pressure in the back and venous hypertension in the lower legs, causing swelling of the collateral veins and dilation [2]. Leg ulceration is the end-stage venous disease [3]. A frequently traumatized vein that is no longer functional is an excellent basis for opening the skin barrier and creating an ulceration.

People injecting drugs are at risk of serious infections, such as necrotizing fasciitis, wound botulism, and cutaneous anthrax due to the nature of the substances being injected [4]. Illegal narcotics are often diluted with other toxic substances, whose acidity contributes to accelerated sclerosis of the veins and faster damage to and opening of the ulcers [5]. The most commonly used drug is heroin. 


\section{CASE REPORTS}

\section{Case 1}

A 34-year-old male and heroin addict had suffered from venous ulcers persisting for about three years with occasional and partial closures. The patient repeatedly refused to be hospitalized and made the bandages on the wound by himself. During an examination, it was revealed that, during the last several days, he noticed an increase in the size of the wounds and an unpleasant odor.

The ulcerations were on the medial side of the left lower leg on erythematous bases, $6 \mathrm{~cm}$ in length in the bigger and $2.5 \mathrm{~cm}$ in the smaller (Fig. 1), with a bottom with fibrin patches and pus and with serrated edges. The patient reported continuous intravenous injection of heroin until one year ago, when he began a rehab program and methadone therapy.

After three years of outpatient treatment, the patient finally agreed to be hospitalized. During the hospitalization, intravenous antibiotic therapy, daily dressing, and compression therapy were performed. Blood counts, differential blood counts, and extended laboratory tests were within normal limits.

Doppler ultrasound showed normal arterial circulation, but significant damage to the venous system, reflux of the saphenous-femoral mouth, remnants of the post-thrombotic process, and significantly dilated and branched $v$. saphena magna and $v$. saphena parva, with numerous perforators at the level of the left lower leg.

The patient was discharged one month after admission with a significant improvement in his dermatological status, and complete closure of the ulcer occurring after six weeks. We believe that the improved clinical picture and the closure of the chronic venous ulcer were due to the changed lifestyle, the intermission of heroin injections in the femoral vein, and the constant care of the wound in the hospital.

\section{Case 2}

A 35-year-old patient addicted to heroin since the age of seventeen visited the dermatological clinic for numerous venous ulcers in the lower extremities. She had a long history of venous ulcers that had only become worse and asked for help several times but was never hospitalized. The wounds were treated badly, without professional treatment and without daily dressings. The patient had been in a bad condition for a long time, and during an examination, it was revealed that she had been in the third month of pregnancy, her gynecologist advised her to undergo an abortion, but she refused and did not return for a fetal checkup. The baby's father was also addicted to drugs and supported her intention not to stay in the hospital and not to visit doctors often. It was almost impossible to do Doppler ultrasound of the blood vessels of the lower extremities due to the poor dermatological status of the lower legs. Extensive, shallow venous ulcers with livid and infiltrated borders (Fig. 2) were present on both lower legs. Therefore, we suspected papillomatosis cutis carcinoides of the foot (Fig. 3), which required a biopsy, but we again encountered rejection. The patient's ulcers were treated and a bandage was applied. The patient did not come for a follow-up examination and refused hospitalization. The patient has had a miscarriage and still has a problem with the open lower leg ulcers.

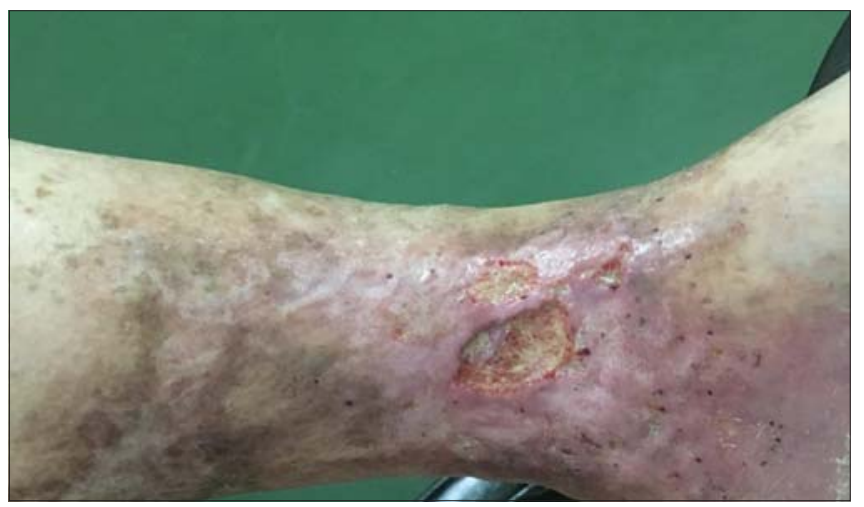

Figure 1: The two ulcers, $6 \mathrm{~cm}$ and $2.5 \mathrm{~cm}$ in size, with a bottom with fibrin patches on erythematous bases and serrated edges.

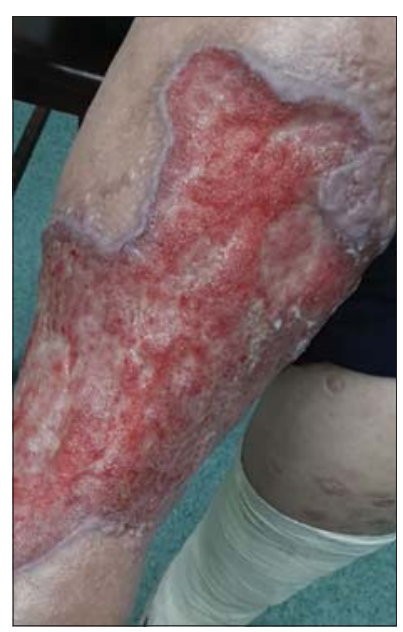

Figure 2: The venous ulcers with livid and infiltrated borders on the right leg. 


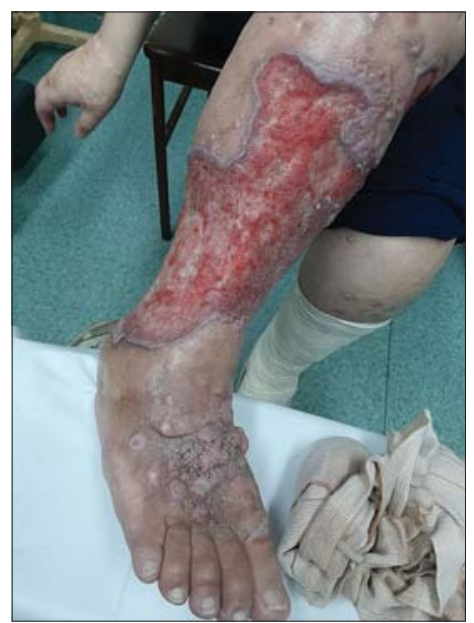

Figure 3: Papillomatosis cutis carcinoides.

\section{DISCUSSION}

In the presence of chronic venous ulcers that last for years, the outcome depends on lifestyle changes. A rehabilitation and hospitalization programs and proper wound care give satisfactory results. Unfortunately, in many young addicted patients, indiscipline and prolonged venous trauma from drug injections lead to greater complications and problems.

The most common problems that we face as medical staff in treating venous ulcers in drug addicts are lack of diligence, often insufficient hygiene, nonadherence to prescribed medical treatment, and refusal of hospitalization, which is probably caused by fear of abstinence. Staff should be careful when treating wounds in these patients as most of them are hepatitis $\mathrm{C}$ positive and some are HIV positive. Working with this type of patients is more complicated than usual as it requires more attention and patience.

Some studies show that problematic drug users also report feelings of worthlessness and shame, and many of them have co-existing mental health problems [6].

\section{CONCLUSION}

It is therefore important to pay attention to informing drug addicts on the consequences of intravenous drug injection and limb care. It is necessary to raise awareness of the importance of treatment and lifestyle changes. The composition and quality of the injected heroin and the duration of the addiction also play a role in the treatment as the degree of sclerosis of the veins depends on these factors. In our case, the patients' mental health and the support and influence of the environment that they have had proved to be crucial. In this context, a multidisciplinary approach in these patients may contribute to the improvement of the venous insufficiency, wound healing, and lifestyle. Quitting drug addiction is a long and arduous process, yet it requires sacrifice, strong will, and work with professionals in the field.

\section{Consent}

The examination of the patient was conducted according to the principles of the Declaration of Helsinki.

\section{REFERENCES}

1. Campbell B. Varicose veins and their management. BMJ. 2006;333:287-92.

2. Miller LM, Gal A. Cardiovascular system and lymphatic vessels. Pathologic Basis of Veterinary Disease. 2017;561-616. e1.

3. Eklöf B, Rutherford RB, Bergan JJ, Carpentier PH, Gloviczki P, Kistner RL, et al. Revision of the CEAP classification for chronic venous disorders consensus statement. J Vasc Surg. 2004;40:1248-52.

4. Coull AF, Atherton I, Taylor A, Watterson AE. Prevalence of skin problems and leg ulceration in a sample of young injecting drug users. Harm Reduct J. 2014;11:22.

5. Williams AM, Southern SJ. Conflicts in the treatment of chronic ulcers in drug addicts: Case series and discussion. Br J Plast Surg. 2005;58:997-9.

6. Rahim M, Patton R. The association between shame and substance use in young people: A systematic review. PeerJ. 2015;3:e737.

Copyright by Mirela Vasileva, et al. This is an open access article distributed under the terms of the Creative Commons Attribution License, which permits unrestricted use, distribution, and reproduction in any medium, provided the original author and source are credited. Source of Support: Nil, Conflict of Interest: None declared. 